\title{
QUALITY INDICATORS TO ASSESS A COLORECTAL CANCER PREVENTION PROGRAM
}

Victoria Serra-Sutton

Catalan Agency of Health Information, Assessment and Quality, CIBER Epidemiología y Salud Pública Barcelona

Carmela Barrantes Serrano

University Hospital Vall Hebrón, Preventive Medicine Service Barcelona (Spain)
Mireia Espallargues Carreras

Catalan Agency of Health Information, Assessment and Quality and CIBER Epidemiología y

Salud Pública Barcelona

Objectives: The aim of this study was to implement a set of indicators to assess the quality of care of a new healthcare model for prevention of colorectal cancer in a high-risk population.

Methods: Information was obtained retrospectively from electronic clinical records, review of documentation, and a survey. The high-risk clinic for colorectal cancer was created in Barcelona (Spain) in 2006. All users at greater risk of colorectal cancer assessed through the new healthcare model were included. Twenty-one indicators were computed using defined formulas and standards. Logistic regression models were computed to analyze factors related to adherence to the screening and surveillance prevention strategies.

Results: A total of 1,275 users were included. Eight of seventeen indicators reached the quality standard ( 80 percent structure, 50 percent process, and 17 percent outcome), whereas four indicators did not have a previously defined standard. The overall adherence to the screening and surveillance program was 67 percent. Users aged 59 and older had almost two times greater probabiblity (95 percent confidence interval $[\mathrm{Cl}], 1.3-3.1$ ) of adherence than younger users; users with surveillance colonoscopies presented a 7.4 times (95 percent $\mathrm{Cl}, 4.6-11.7$ ) greater probability of adherence than those with screening colonoscopies.

Conclusions: The indicators have been shown to be feasible and valid tools to identify areas of improvement in this new model, such as information systems, continuity of care, and communication among professionals. Because this was the first time these indicators were applied to assess the high-risk clinic for colorectal cancer, further implementation is required to improve the interpretability of results.

Keywords: Quality indicators, Colorectal cancer, Health technology assessment, Adherence, Preventive medicine

Most prevention programs for colorectal cancer (CRC) are aimed at medium-risk populations and are included in population-based prevention strategies. The main inclusion criteria in this type of program is age (aged 50 and older without personal or family history of colorectal adenomas or CRC) $(1,2)$. There is another risk group, made up generally of individuals at increased risk of developing $\mathrm{CRC}$, which includes the following criteria: (i) individuals who carry mutations in certain genes, (ii) individuals with a family history of CRC, and (iii) individuals with a history of high-risk colorectal adenoma-

The research team thanks Antoni Castells, director of the Institute of Digestive and Metabolic Disorders at the Hospital Clinic de Barcelona, for his collaboration in collecting and categorizing study variables, for his input into the healthcare model of the High Risk Clinic for Colorectal Cancer (HRC-CRC), for his help in identifying risk profiles of users, and for his comments on a previous version of this manuscript. We also thank Maria Pellice and Teresa Ocaña from the Gastroenterology Service for their help in recoding variables and for providing the necessary information for the collection of data for some indicators. Our thanks also go to Montserrat Milà, Imma Garrell, Josep Maria Peri, Leticia Moreira, Maria Dolores Giráldez, Miriam Cuatrecases, Sergi Castellvi-Bel, and María López-Cerón, for their collaboration in the project. From the Catalan Agency for Health Information, Assessment and Quality, we also thank Silvia Lopez Aguilar for tous polyps. Individuals in the group of carriers of mutations in specific genes represent between 3 percent and 5 percent of all patients diagnosed with CRC.

In 2006, the High-Risk Clinic for Colorectal Cancer (HRCCRC) $(3 ; 4)$, a new healthcare model for the prevention of CRC in a high-risk population, was created at the Hospital Clinic

her help in reviewing the database and in defining the complex syntax for creating variables; Antoni Parada for his exhaustive literature searches to identify similar studies that also assessed models/programs for the prevention of CRC in populations at high risk based on indicators; and Olga Martinez for her help in field work. Finally, we thank Elena Dopino for the editing of the final English manuscript. This project was partially funded by the Quality Plan of the Spanish Ministry of Health 2009 and also consitutes a technical consultation from the Catalan Department of Health. Carmela Barrantes Serrano is a resident of Prevention Medicine and Public Health at the Vall Hebron University Hospital in Barcelona. This article is part of her Master of Public Health thesis defended in September 2010 at the Pompeu Fabra University. We are also grateful to Carme Borrell and Andreu Segura as evaluators of the thesis research protocol, which improved the feasibility of the proposed dissertation and its methodological justification. We also thank Xavier Castells for his comments on a draft document as a thesis evaluator before final defense. 
of Barcelona under the coordination of the Institute of Digestive and Metabolic Disorders. The Hospital Clinic of Barcelona is a reference, community, level 1, public teaching hospital in Catalonia with renowned capabilities, both nationally and internationally, in healthcare, research, innovation, and quality teaching. Catalonia is one of the seventeen autonomous regions in Spain with a regional administration and autonomous government and parliament. The HRC-CRC was funded by the Catalan Department of Health with the aim of implementing prevention strategies in populations at greater risk of CRC (individuals with a hereditary or individual predisposition to colorectal cancer such as Lynch syndrome, familial adenomatous polyposis, or advanced colorectal adenomas). The HRC-CRC is staffed with health professionals from the fields of gastroenterology, biochemistry and molecular genetics, pathology, clinical psychology, nursing, gastrointestinal surgery, oncology, and family and community medicine. The roles of this new healthcare model/program included the following main areas of healthcare: (i) to identify individuals at increased risk of developing CRC (mainly individuals referred from primary health care according to evidence-based criteria in relation to CRC risk factors of increased risk or other specialized services from their own hospital or from other hospitals; patients and users also access the program on their own initiative; (ii) to establish the risk of CRC in users with personal risk factors and/or a family history of CRC; (iii) to propose the most appropriate screening and surveillance prevention strategies; (iv) to carry out genetic counseling in hereditary forms of CRC, genetic testing, and psychological assessment; (5) to carry out additional endoscopic and radiological procedures for prevention, diagnostic; and/or therapeutic aims.

In a first phase of implementation of the HRC-CRC, the Hospital Clinic of Barcelona, pending the incorporation of a secondary hospital, assumed the role of secondary and tertiary care provider, and was linked to two primary care centers, which took on the primary level functions of this new organizational model for the prevention of CRC. Tertiary level functions of this model consist mainly in the implementation of measures for genetic counseling and testing, and the performance of complex surgical or endoscopic procedures (e.g., Lynch syndrome). Secondary level functions include the management of high-risk forms of CRC requiring colonoscopies with a periodicity of less than 5 years (e.g., familial CRC) and the performance of conventional and therapeutic endoscopic procedures. Finally, functions at the primary care level consist in identifying populations at greater risk of CRC and the followup of users on the prevention program with intervals of over 5 years between colonoscopies (e.g., patients with non-advanced adenomas).

Although the activities (clinical practice) of the HRC-CRC are evidence-based, to the best of our knowledge, no assessment of the quality of care and the benefits/risk of the prevention program on the user's health have been carried out $(4 ; 5)$. To date, no published study has been identified that evaluates the quality of care of a CRC prevention program in a high-risk population taking into account all the healthcare processes involved. The primary objective of this study was to implement a set of indicators to assess the quality of care of a new healthcare model for the prevention of colorectal cancer in a high-risk population (at baseline and independently). Furthermore, as a secondary objective, factors related to adherence to screening and surveillance prevention strategies in this model were analyzed. Several studies have evidenced that demographic factors such as user gender, age, and health status are related to their adherence to screening and surveillance prevention strategies $(6 ; 7)$. The assessment of factors relating to adherence to this type of strategies in the HRC-CRC was considered relevant to assess the construct validity of three proposed indicators measuring patient-centered care.

\section{METHODS}

A retrospective observational and descriptive study was carried out. The study population included all users assessed at the HRC-CRC between January 1, 2006, and May 12, 2010. Patients with advanced colorectal adenoma, hereditary polyposis syndromes, CRC, and healthy users at increased risk (family members of patients with CRC or hereditary syndromes) aged 18 and older were included in this study $(3 ; 4)$.

\section{Development of Quality Indicators}

In a previous study, thirty indicators were developed to assess the quality of care of the HRC-CRC or other prevention programs in Catalonia/Spain (8). A conceptual framework for the assessment of these programs and the definition of indicators was proposed. This framework included the main activities (process of care) of the new prevention program from the perspective of Donabedian for quality assessment (including the measure of the improvement of health services results and also the structure and process of healthcare). Five domains were also proposed in the framework to measure the quality of healthcare (clinical effectiveness, continuity of health care, access, safety, and patient-centered care).

A literature review was performed to identify published studies that assessed CRC prevention programs in a high risk population using quality indicators and their conceptual frameworks. Two consensus meetings were held with twelve experts from the Hospital Clinic of Barcelona and the Hospital Costa del Sol in Malaga (including the fields of gastroenterology, nursing, primary care medicine, molecular genetics, pathology, clinical psychology, medical oncology, management, and health services research). Experts were asked to propose indicators following the above-mentioned conceptual framework. Indicators were arranged in panels by the moderator of the consensus meeting (metaplan technique) according to the defined framework. All proposed indicators were voted by the experts in a final round. Stickers were placed on the indicators by each expert when deemed important or feasible. Two focus groups that 
included users from the HRC-CRC were also held to obtain additional indicators that had not been mentioned by the experts. The research team developed cards for each indicator, including the title of the indicator, formula, description and justification, sources of information to obtain the indicators, and quality standard.

Final consensus of indicators (cards) was defined by applying the Delphi technique, with the participation of fifty-three experts from different centers, regions in the Spanish Health System, and the same multidisciplinary profiles included in consensus meetings. Experts were asked to vote on the importance and feasibility of (on a scale of 1 to 10 , where 1 was minimum and 10 was maximum) as well as their agreement with each proposed indicator (fully agree, agree with modifications, or fully disagree) (8). Experts in this final consensus were asked to propose a quality standard for each indicator (the standard was calculated using the mean value obtained from all the experts).

\section{Indicators, Variables, and Sources of Information in the Phase of Implementation}

Before collecting the data to implement the indicators, the research team reviewed each indicator taking into account a preliminary revision of available data, the feasibility to calculate each indicator in the study schedule, simplicity of the formula computation, and those indicators that applied to the majority of the study population. Of the thirty proposed indicators, twenty were selected for implementation and one additional indicator was proposed that included a composite of two previous indicators (global adherence to the prevention program-screening and surveillance colonoscopies); see Supplementary Material, which can be viewed online at www.journals.cambridge.org/thc2013089 (6).

The necessary information for calculating each indicator, together with user demographic and clinical variables at the HRC-CRC, were collected, including gender; age $(<45,48-59,>59)$; type of prevention strategy (a) screening colonoscopy, which suggests an examination of the colon, following evidence-based clinical criteria; if abnormal areas are detected, tissue can be removed and examined to determine if there is presence of disease; (b) surveillance colonoscopy, which suggests a periodical examination of the colon, following evidence-based clinical criteria, after the detection or removal of disease in the colon) ${ }^{1}$; (c) personal risk at the moment of inclusion in the study (advanced colorectal adenoma, polyposis syndrome, CRC, healthy high-risk relative); and site of referral (other hospitals, other intra-hospital services, primary care, and user's own initiative).

The primary source of information for calculating the process and outcome indicators was the information-based clinical records of the HRC-CRC (Progeny). In the case of structure

\footnotetext{
${ }^{1}$ Adapted from definitions included in the National Cancer Institute, http://www.cancer.gov [consulted 17/07/2012].
}

indicators, clinical and management documentation was consulted. Additional information was collected through questionnaires sent by email to professionals or collected in face-to-face interviews at the HRC-CRC by the research team.

\section{Statistical Analysis}

Twenty-one indicators were computed according to a previously defined formula, and results obtained were compared with defined quality standards ( $0-100$ percent) (8). For some composite indicators, global and partial compliance was described. Star graphs were computed to present a visual differentiation between the defined quality standard and the level of compliance.

Donabedian and other authors consider that outcomes of health services are mainly related to the structure and process of healthcare as well as to patient/user characteristics $(9 ; 10)$. To apply this perspective, the research team revised the proposed indicators and selected adherence to the screening and surveillance prevention program as an outcome indicator for multivariate analysis. The reasons for selecting this indicator were mainly due to the fact that it applied to the majority of the patients/users assessed in the new organizational model (ensuring an effective number of cases in the numerator and denominator of the formula to compute adherence). In addition, this indicator was also considered an intermediate-term outcome (in contrast with the global effectiveness of the program [diagnosis of colorectal cancer in early stages], which represented a small number of cases and needed a longer follow-up period to obtain a robust measurement). Most of the variables related to adherence to screening and surveillance prevention strategies in colorectal cancer used in the present study have been previously reported in other published studies.

To identify factors related to global adherence to screening and surveillance prevention strategies, multiple regression models were computed using global adherence as the dependent variable and user demographic, health status, and healthcare process factors that showed statistical significance on bivariate analysis $(p<.05)$ or those that were hypothesized to be conceptually relevant (as described previously in the variables section) as independent variables. Each logistic model was adjusted by gender and age differences. The SPSS package was used for the statistical analysis.

\section{RESULTS}

\section{Characteristics of Patients and Users and Some Aspects of the Healthcare Process}

The initial database contained information on 1,607 users; 311 users that had been included in the HRC-CRC before January 1,2006 (data at the start of the new healthcare model), 4 users that were under the age of 18 , and 17 users without high risk of CRC in the database, were excluded from the study. Of the total sample, 54 percent were women, and 34 percent were over the age of 59 (Table 1). In addition, 63.1 percent of users were seen due to a family history of CRC (screening prevention strategy), 
Table 1. Demographic, Health Status, and Process Characteristics of Patients in the HRC-CRC in the Period from 2006 to $2010(n=1,275)$

\begin{tabular}{|c|c|c|}
\hline Variables & N & $\%$ \\
\hline \multicolumn{3}{|l|}{ Gender } \\
\hline Women & 692 & 54.3 \\
\hline Men & 583 & 45.7 \\
\hline Missing values & - & - \\
\hline \multicolumn{3}{|l|}{ Age } \\
\hline$<45$ years old & 385 & 30.2 \\
\hline $46-58$ years old & 425 & 33.3 \\
\hline$>59$ years old & 434 & 34.0 \\
\hline Missing values & 31 & - \\
\hline \multicolumn{3}{|l|}{ Personal risk status } \\
\hline Advanced colorectal adenoma ${ }^{a}$ & 589 & 46.2 \\
\hline Polyposis syndrome ${ }^{b}$ & 107 & 8.4 \\
\hline Colorectal cancer & 163 & 12.8 \\
\hline Healthy relative at high risk ${ }^{c}$ & 416 & 32.6 \\
\hline Missing values & - & - \\
\hline \multicolumn{3}{|l|}{ Endoscopic prevention strategy } \\
\hline Screening & 805 & 63.1 \\
\hline Surveillance & 399 & 31.3 \\
\hline Missing values & 71 & - \\
\hline \multicolumn{3}{|l|}{ Referral to the coordinating service } \\
\hline From primary care & 548 & 43.0 \\
\hline Intra-hospital units & 363 & 28.5 \\
\hline Patient's own initiative & 136 & 10.7 \\
\hline Other hospitals & 45 & 3.5 \\
\hline Other prevention programs & 24 & 1.9 \\
\hline Missing values & 159 & - \\
\hline \multicolumn{3}{|l|}{ Endoscopic tests carried out } \\
\hline Includes baseline and follow-up & 413 & 32.4 \\
\hline Only includes baseline & 200 & 15.7 \\
\hline Includes baseline but follow-up does not apply & 193 & 15.1 \\
\hline Baseline does not apply & 408 & 32.0 \\
\hline Missing values & 61 & - \\
\hline
\end{tabular}

aLesions $\geq 10 \mathrm{~mm}$, with villous component or high grade dysplasia.

bMutations in certain genes.

cHealthy relative at risk: the user has been included in the HRC-CC but a polyposis syndrome or high-risk colorectal adenoma or CRC have not been identified.

HRC-CC, high-risk clinic for colorectal cancer.

46.2 percent presented a high-risk colorectal adenoma at the time of the study, 63.2 percent had had at least one colonoscopy, and 32.4 percent had had two colonoscopies (at baseline and at follow-up). A total of twelve professionals provided information on some indicators related to the quality of care in the HRCCRC.
Compliance of Indicators with Quality Standards

Structure indicators that reached the quality standard were: availability of information-based clinical records in the program (100 percent), access to an accredited and certified molecular genetics laboratory, and availability of prevention campaigns aimed at users and patients in the HRC-CRC (100 percent) (Figure 1a). Even though an evidence-based protocol and a clinical practice guideline were available at the HRC-CRC, the indicator that assessed the existence of a clinical protocol reached an overall compliance of 73.9 percent. The specific aspects of the protocol that did not meet quality standards were the failure to include: all activities with scientific evidence, algorithms (referral to clinical psychology service or molecular genetics), and the collaboration of all professional profiles in the development of such protocol. The level of implementation of the clinical protocol by professionals reached a compliance level of 83.3 percent.

Figure $1 \mathrm{~b}$ shows compliance levels achieved by the process indicators. The rate of users referred to the program was 43.0 percent from primary care and 28.5 percent from other in-patient departments or services (data not shown). On the other hand, 1.9 percent of users with a possible polyposis syndrome needing molecular genetic testing were referred to clinical psychology. In families with hereditary CRC with an associated known mutation, a presymptomatic diagnosis through genetic testing was carried out in 56 percent of relatives at risk. In relation to the waiting time to obtain the results of the genetic test, 33 percent of users who had undergone this test obtained the results 12 months after the time of request. Finally, regarding the comprehension of the information given to users in the program, only 19 percent had completed a comprehension questionnaire and these corresponded to users who had been visited by the nurse in the program.

Regarding outcome indicators, the rate of complications during colonoscopies was 0.6 percent (data not shown), while the rate of early-stage CRC diagnosis was 55.6 percent (as a proxy of the global effectiveness of the program). Adherence to screening and surveillance prevention strategies achieved the agreed standard for the surveillance strategy (mainly applied to patients with advanced colorectal adenomas; 91.2 percent), whereas for the screening strategy, the level of compliance was 51.7 percent, below the agreed quality standard ( 80 percent) (Figure 1c). The compliance to global adherence was 67.3 percent. User satisfaction with the care provided was measured in 19 percent of the 599 users seen between 2008 and 2010 (period in which the collection of information started).

Overall, eight of seventeen indicators reached the previously defined quality standard (80 percent in the case of structure, 50 percent of process, and 17 percent of outcome indicators). Moreover, 4 indicators were computed but did not have a previously defined standard. When taking into account the quality domain measured, 40 percent of the indicators that measured clinical effectiveness achieved the standard (2/5), 100 percent of the indicators that measured accessibility achieved the standard 
Serra-Sutton et al.

(a)

4

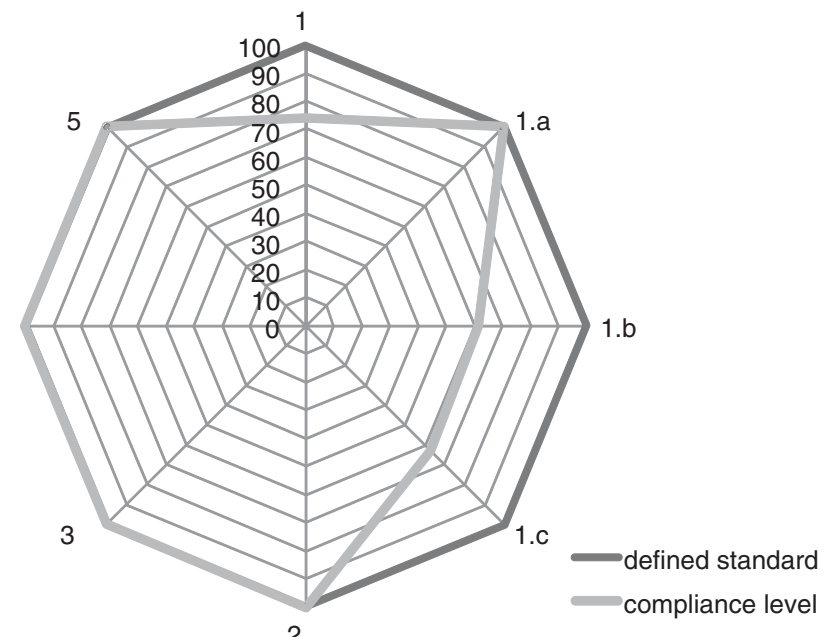

(b)

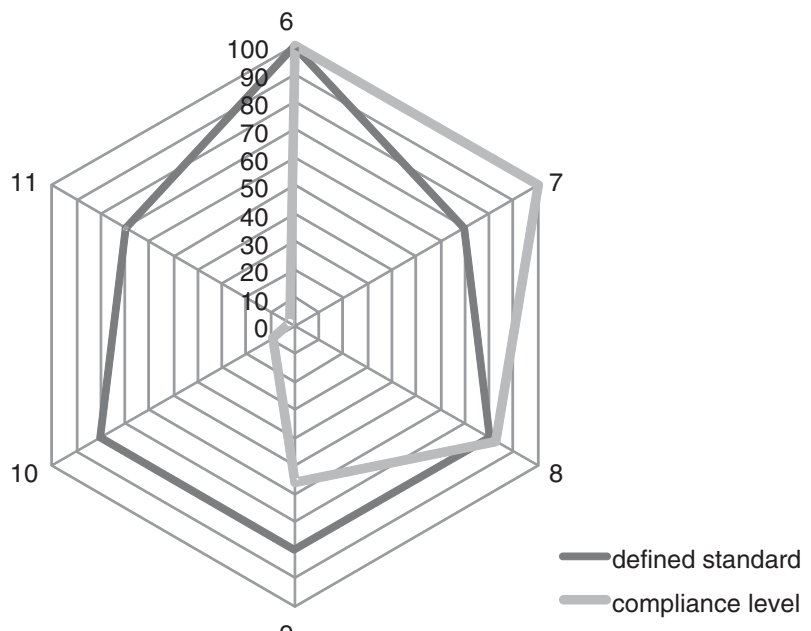

9

(c)

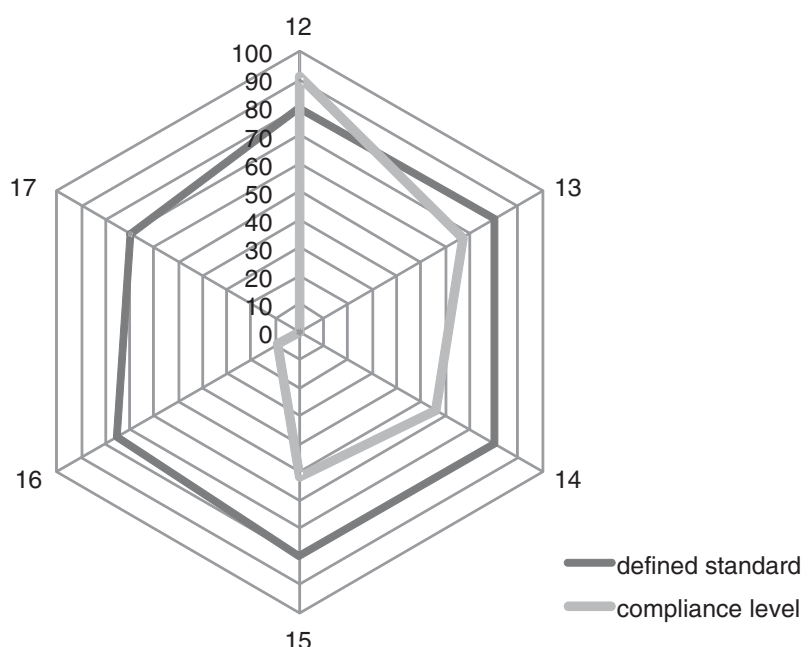

Figure 1. Level of compliance of indicators with defined quality standards. a: Level of compliance of structure indicators. 1. Availability of a multidisciplinary clinical evidence-based protocol; la. Protocol with recommendations, decision tools; 1b. Protocol with evidence- based activities; Ic. Multidisciplinary profile of authors of the protocol; 2. Access to a certified and accredited molecular genetic testing laboratory; 3. Availability of a side-viewing duodenoscopy for screening of patients with polyposis syndrome; 4 . Availability of prevention strategies of colorectal cancer in a high-risk population for users and patients; 5 . Existence of information-based clinical records. $\mathrm{b}$ : Level of compliance of process indicators. 6. Availability of a colonoscopy quality program; 7. Rate of indication of dietetic and nutritional needs assessment; 8 . Level of implementation of the clinical protocol; 9 . Presymptomatic diagnosis of users with hereditary colorectal cancer with genetic testing; 10. Administration of a comprehension questionnaire to users; 11. Rate of indication of psychological assessment of users in the program. c: Level of compliance of outcome indicators. 12. Adherence to surveillance preventive strategies in patients with colorectal adenomas; 13. Global adherence to screening and surveillance preventive strategies in users at high risk of colorectal cancer; 14. Effectiveness of the program (diagnosis of early stage (RC); 15. Adherence to screening preventive strategies in users at high risk of colorectal cancer; 16. Administration of a satisfaction questionnaire to users; 17. Administration of a questionnaire to users to measure the impact of the program on their physical and emotional well-being. 
Table 2. Demographic and Clinical Factors Related to Global Adherence to Screening and Prevention Strategies in Users in the HRC-CRC, 2006-2010: Logistic Regression Analysis of Adherence to Colonoscopies $(n=613)^{a}$

\begin{tabular}{|c|c|c|}
\hline Variable & OR (Cl 95\%) raw & OR (CI 95\%) adjus \\
\hline \multicolumn{3}{|l|}{ Gender } \\
\hline Women & $1^{c}$ & $j^{c}$ \\
\hline Men & $1.4(1.0-2.0)$ & $1.3(0.9-1.9)$ \\
\hline \multicolumn{3}{|l|}{ Age } \\
\hline$<45$ years old & $j^{c}$ & $j^{c}$ \\
\hline $46-58$ years old & $1.5(0.9-2.4)$ & $1.5(0.9-2.4)$ \\
\hline$>59$ years old & $2.0(1.3-3.2)$ & $2.0(1.3-3.1)$ \\
\hline \multicolumn{3}{|l|}{ Personal risk status } \\
\hline Healthy relative at high risk & $1^{c}$ & $1^{c}$ \\
\hline Advanced colorectal adenoma & $3.8(2.6-5.7)$ & $3.6(2.4-5.3)$ \\
\hline Colorectal cancer & $7.9(3.9-15.8)$ & $7.0(3.5-14.1)$ \\
\hline Polyposis syndrome & $14.6(6.5-33.3)$ & $14.4(6.3-32.9)$ \\
\hline \multicolumn{3}{|l|}{ Endoscopic prevention strategy } \\
\hline Screening & $1^{c}$ & $1^{c}$ \\
\hline Surveillance & $7.7(4.9-12.1)$ & $7.4(4.7-11.7)$ \\
\hline \multicolumn{3}{|l|}{ Referral to the coordinating service } \\
\hline Patient's own initiative & $1^{c}$ & $1^{c}$ \\
\hline Other hospitals & $2.0(0.6-5.9)$ & $2.0(0.7-6.3)$ \\
\hline Intra-hospital units & $1.7(0.9-3.3)$ & $1.4(0.7-2.7)$ \\
\hline Primary care & $0.5(0.3-0.9)$ & $0.4(0.2-0.8)$ \\
\hline
\end{tabular}

presented a 14.4 times (95 percent CI, 6.3-32.9) greater probability of complying with scheduled follow-up examinations than those relatives with a high-risk profile for CRC. Users referred by other hospitals were 2 times more likely to adhere to the program than those who came to the HRC-CRC on their own initiative, although differences were not statistically significant. Finally, users who had undergone surveillance colonoscopies were 7.4 (95 percent CI, 4.6-11.7) more likely to adhere to the program than those who had had screening colonoscopies.

\section{DISCUSSION}

To the best of our knowledge, this study is the first to evaluate the quality of care and the areas of improvement of a new organizational model for the prevention of CRC in an increased-risk population, taking into account the entire care process and a multidisciplinary perspective. Some publications have assessed the quality of care of CRC prevention programs; however, most of these studies were conducted in medium-risk populations and focused only on one or two aspects of the healthcare process. For example, one study evaluated the quality of colonoscopies in eight endoscopic services and included indicators such as the existence of informed consent, medication before colonoscopy, the availability of complete colonoscopy, or complications (11). Another study developed fifteen indicators of quality of care to assess colon surgery in patients with CRC (e.g., the appropriateness of adjuvant therapy and postoperative surveillance strategies) but only assessed two specific aspects of the organizational model of the HRC-CRC (colonoscopies and surgery) (12). Finally, some studies that included patient-centered indicators such as satisfaction with prevention strategies and adherence to screening colonoscopies of users and their relatives were also carried out in medium-risk populations (users aged 50 and older without individual or family increased risk) $(13 ; 14)$.

Regarding the program's patient safety, the rate of complications during colonoscopies was relatively low ( 0.6 percent) but greater than the published standards in medium-risk populations $(0.1$ to 0.3 percent $)(15 ; 16)$. Nevertheless, greater-risk and medium-risk populations are not strictly comparable in terms of clinical characteristics and type of complications. It is possible that in high-risk populations, the incidence of complications is slightly greater due to the need for therapeutic maneuvers that are associated with increased risk of complications. It should be mentioned that an increased waiting time for genetic testing results in patients with a probable hereditary syndrome may have an impact on their psychological well-being. This impact was mentioned in a qualitative study on users of the HRC-CRC carried out in a previous phase of the project for the definition of indicators as well as in other published studies on perceived outcome measures in genetic counseling and testing $(8 ; 17 ; 18)$. In future studies, complications from all the prevention interventions of a given program should be described, especially those that may be more difficult to measure such as the psychological 
or physical discomfort caused by the program (colonoscopies, genetic and molecular testing, preventive surgery, etc.).

Regarding adherence to screening and surveillance prevention strategies, the quality standard was met in the surveillance strategy (patients with high-risk colorectal adenomas), but not in the screening strategy. This lower adherence in healthy relatives could be due possibly to a lower user perception of CRC risk than users with a diagnosis of polyposis syndrome, advanced colorectal adenoma or CRC. Other published studies show similar results regarding factors, such as gender, age, and risk of $\mathrm{CRC}$, explaining differences in the adherence to screening and surveillance prevention strategies $6 ; 7 ; 14 ; 19 ; 20)$. The lower adherence in healthy relatives with increased risk of CRC might also be due to multiple factors such as individual psychosocial characteristics, relationship with the doctor, family and social environment, and lower perceived risk (21-23).

More and more often, current medical actions are aimed at prevention, as part of community programs for secondary prevention but also as part of many clinical activities, being the balance between the benefits and risks of these activities in many cases uncertain (24). In recent publications, several authors have criticized the heavy reliance on health prevention activities (2527). The present study failed to demonstrate strictly the benefits of the prevention program in terms of health gains for the population, for instance the impact of the new organizational model (the HRC-CRC) to reduce the incidence of CRC or its mortality rate. In relation to the overall effectiveness of the program, a lower percentage than expected of patients with early-stage CRC (0-I) were attended in the HRC-CC. Longer follow-ups should be performed to analyze the capability of the program to detect early-stage CRC patients. As said before, at present, users are mainly referred to the HRC-CRC from other hospital services, which may explain the increased severity of the patients' disease. Indicators that were not implemented in the present project, such as colorectal cancer that is diagnosed between two colonoscopies (interval CRC) or the overall effectiveness of the program to detect CRC, as well other patient-perceived indicators such as the previously mention impact of the program on the psychological and physical well-being of users, need to be measured in future studies.

Because the indicators have been applied in a single center and at baseline, this could lead to limitations in the interpretation of compliance with the quality standards. The fact that there are no published standards for each of the indicators, or the absence of consensus on some of them, hampers the interpretation of results. Further assessment of the HRC-CRC or the implementation of indicators in other prevention programs to assess the quality of care and to confirm the robustness and validity of the indicators is therefore required $(28 ; 29)$. In this study, modifications were made to improve the accuracy and validity of indicators, in terms of specification of the formula, description/justification, or even the standard if identified in the literature.

\section{Limitations of the Study}

Although the assessment of the quality of care based on the use of indicators appears to be relatively easy to perform, it has required a complex review of data quality. The retrospective nature of study and the use of computerized clinical records as the main source of information have hampered the processing of data for analysis and the calculation of some indicators. This database was not developed for research purposes, but for the clinical management of users. However, it was possible for the first time to assess data quality and completeness from information-based clinical records. Finally, it should be mentioned that the health of users changes over time, complicating the definition of their risk profile for statistical analysis. We used the most definitive diagnosis at the time of analysis, but users could have gone from a "healthy profile" to a "polyposis syndrome" in a short time, a fact that may have affected the accuracy of the association between adherence and CRC risk profiles.

\section{CONCLUSION}

Several indicators have been implemented to assess the quality of care in the HRC-CRC. They have demonstrated their feasibility and construct validity to identify areas of improvement of this new model, such as information systems, continuity of care, and communication among professionals. This is one of the first initiatives to have externally evaluated a CRC prevention program in a high-risk population in Catalonia. The fact that there are no published standards for each of the indicators makes the study an innovative assessment tool. However, further assessment or implementation of the indicators in other centers and in the HRC-CRC is still required to improve the interpretability of the results.

\section{SUPPLEMENTARY MATERIAL}

Supplementary Material:

www.journals.cambridge.org/thc2013089

\section{CONTACT INFORMATION}

Victoria Serra Sutton, Sociologist, PhD, (vserra@aatrm. catsalut.cat), Catalan Agency for Health Research and Quality and CIBER Epidemiología y Salud Pública, Barcelona, Spain

Carmela Barrantes Serrano, MD, University Hospital Vall Hebrón, Preventive Medicine Service, Barcelona, Spain

Mireia Espallargues Carreras, MD, PhD, Catalan Agency for Health Research and Quality and CIBER Epidemiología y Salud Pública, Barcelona, Spain

\section{CONFLICTS OF INTEREST}

All authors report a grant to their institution from Quality Plan, Spanish Ministry of Health 2009. 


\section{REFERENCES}

1. Ribes J, Navarro M, Cleries R, et al. Survival for eight major cancers and all cancers combined for European adults diagnosed in 1995-99: Results of the EUROCARE-4 study. Lancet Oncol. 2007;8:773-783.

2. Working Group of the clinical practice guideline for colorectal cancer prevention. Clinical practice guideline. Colorectal Cancer Prevention. Update. Barcelona: Asociación Española de Gastroenterología, Sociedad Española de Medicina de Familia y Comunitaria y Cochrane Iberoamericano; 2009. [guideline in Spanish].

3. Balaguer F, Ocaña T, Garrell I, Ferrández A. High risk clinics: A new concept in colon and rectum cancer prevention. Med Clin (Barc). 2008;131:382-386. [article in Spanish].

4. High Risk Clinic Colorectal Cancer. Clinical High Risk of Colorectal Cancer: A new model for the prevention of colon cancer. Manual d'actuació: Hospital Clínic de Barcelona, Consorci d'Atenció Primària de Salut de l'Example, Àrea Bàsica de les Corts i Hospital General de Vic. Barcelona; 2008. [clinical protocol in Catalan].

5. Castells X, Sala M, Ascunce N, et al. Description cancer screening in Spain. DESCRIC Project. Barcelona: Plan de Calidad para el Sistema Nacional de Salud del Ministerio de Sanidad y Política Social. Ministerio de Ciencia e Innovación. Agència d' Avaluació de Tecnologia i Recerca Médiques de Catalunya; 2007. Informes de Evaluación de Tecnologías Sanitarias, AATRM n. ${ }^{\circ}$ 2006/01. [report in Spanish].

6. Griffin JM, Burgues D, Vernon SW, et al. Are gender differences in colorectal cancer screening rates due to differences in self-reporting? Prev Med. 2009;49:436-441.

7. Denberg TD, Melhado TV, Coombes JM, et al. Predictors of nonadherence to screening colonoscopy. J Gen Intern Med. 2005;20:989-995.

8. Serra-Sutton V, Barrionuevo L, Herdman M, et al. Development of quality indicators to assess colorectal cancer prevention programs in a high risk population. Barcelona: Plan de Calidad para el Sistema Nacional de Salud, Ministerio de Sanidad y Política Social. Ministerio de Ciencia e Innovación. Agència d' Avaluació de Tecnologia i Recerca Médiques de Catalunya; 2010. Informes de Evaluación de Tecnologías Sanitarias, AATRM núm. 2009/01. [report in Spanish].

9. Donabedian A. The quality of medical care: How can it be assessed? JAMA. 1988;260:1743-1748.

10. Starfield B. Basic concepts in population health and health care. J Epidemiol Community Health. 2001;55:452-454.

11. Sánchez del Río A, Baudet JS, Naranjo A, Campo R, et al. Development and validation of quality standards for colonoscopy. Med Clin. 2010;134:49-56. [article in Spanish].

12. Gagliardi A, Simunovic M, Langer B, et al. Development of quality indicators for colorectal cancer surgery using a 3 step modified Delphi approach. Can J Surg. 2005;48:441-452.

13. Sánchez del Río A, Campo R, Llach J, et al. Patient satisfaction in gastrointestinal endoscopy: Results of a multicenter study. Gastroenterol Heaptol. 2008;31:566-571. [article in Spanish].

14. Bujanda L, Catells A, Llor X, et al. Low adherence to colonoscopy in the screening of first-degree relatives of patients with Colorrectal cáncer. Gut. 2007;56:1714-1718.

15. Crispin A, Birkner B, Munte A, et al. Process quality and incidence of acute complications in a series of more than 230000 outpacient colonoscopies. Endoscopy. 2009;41:1018-1025.

16. Singh H, Penfold RB, De Coster C, et al. Colonoscopy and its complications across a Canadian regional health authority. Gastrointest Endosc. 2009;69:665-671.

17. Payne K, Nicholls S, McAllister M, et al. Outcome measurement in clinical genetic services: A systematic review of validated measures. Value Health. 2008;11:497-508.

18. Watkins KE, Way CY, Fiander JJ, et al. Lynch syndrome: Barriers to and facilitators of screening and disease management. Hered Cancer Clin Pract. 2011;9:8.

19. Mack LA, Cook LS, Temple WJ, et al. Colorectal cancer screening among first-degree relatives of colorectal cancer patients: Benefits and barriers. Ann Surg Oncol. 2009;16:2092-2100.

20. Christie J, Nassisi D, Wilets I, et al. Assesing endoscopic colorectal screening adherence in emergency department population. $J$ Natl Med Assoc. 2006;98:1095-1101.

21. Taouqi M, Ingrand I, Beauchant M, Migeot V, Ingra P. Determinants of participation in colonoscopic screening by siblings of colorectal cancer patients in France. BMC Cancer. 2010;10:355.

22. Ruthotto M, Papendorf F, Wegener G, et al. Participation in screening colonoscopy in first-degree relatives from patients with colorectal cancer. Ann Oncol. 2007;18:1518-1522.

23. Cottet V, Pariente A, Nalet B, et al. Low compliance with colonoscopic screening in first-degree relatives of patients with large adenomas. Aliment Pharmacol Ther. 2006;24:101-109.

24. Raffle A, Muir Gray JA. Screening: Evidence and practice. New York: Oxford University Press; 2009.

25. Gérvas J, Starfield B, Heath L. Is clinical prevention better than cure? Lancet. 2008;372:1997-1999.

26. Starfield B, Hyde J, Gérvas J, Health I. The concept of prevention: A good idea gone astray? J Epidemiol Community Health. 2008;62:580583.

27. Permanyer G. ¿Son valores antagónicos la prevención y la compasión? Med Clin (Barc). 2010. doi:10.1016/jmedcli.2009.11.026.

28. Campbell SM, Braspenning J, Hutchinson A, et al. Improving the quality of health care: Research method used in developing and applying quality indicators in primary care. BMJ. 2003;326:816-819.

29. Wollersheim H, Hermens R, Hulscher M, et al. Clinical indicators: Development and applications. Neth J Med. 2007;65:15-22. 